\title{
Cardiovascular outcomes in adults with migraine treated with eptinezumab for migraine prevention: pooled data from four randomized, double-blind, placebo- controlled studies
}

Timothy R. Smith ${ }^{1 *}$, Egilius L. H. Spierings ${ }^{2}$, Roger Cady ${ }^{3}$, Joe Hirman ${ }^{4}$, Anders Ettrup ${ }^{5}$ and Vivienne Shen ${ }^{3}$

\begin{abstract}
Background: Patients with migraine have an increased relative risk of cardio- and cerebrovascular events, and some migraine treatments may exacerbate this risk. The primary objective of this analysis was to determine whether the rate of cardiovascular adverse events was higher for patients with migraine treated with the migrainepreventive eptinezumab, compared with patients receiving placebo.

Methods: Cardiovascular outcomes in patients with migraine were pooled across four clinical trials (phase 1b, phase 2, and two phase 3 trials) for use of eptinezumab as a preventive migraine treatment for up to 1 year. In all studies, treatment-emergent adverse events (TEAEs) that occurred after the first dose of study treatment (eptinezumab 100 mg, 300 mg, 1000 mg, or placebo) and vital signs were recorded through study completion.

Results: Cardiovascular TEAEs were rare across all four clinical trials, and rates were similar between patients receiving eptinezumab and those receiving placebo. Cardiovascular TEAEs that did occur were mild or moderate in severity; there were no serious adverse events as per FDA definition. Vital signs (systolic blood pressure, diastolic blood pressure, and heart rate) were not meaningfully different across treatment groups over the course of 56 weeks, compared to placebo. Treatment with eptinezumab did not result in significant new or changed cardiovascular medications used concomitantly compared to placebo.
\end{abstract}

Conclusions: In this post hoc analysis of four clinical trials for eptinezumab, doses of $100 \mathrm{mg}, 300 \mathrm{mg}$, and $1000 \mathrm{mg}$ (more than 3 times the highest approved dose) were not associated with clinically relevant changes in vital signs or significant changes in concomitant cardiovascular medication usage, and had low incidences of cardiovascular TEAEs, comparable to placebo.

Trial registration: NCT01772524 (Study 2), 01/21/2013; NCT02275117 (Study 5), 10/27/2014; NCT02559895 (PROMISE-1), 09/25/2017; NCT02974153 (PROMISE-2), 11/28/2016

Keywords: Eptinezumab, Cardiovascular, CGRP, Migraine

\footnotetext{
* Correspondence: tsmith@studymetrix.com

${ }^{1}$ StudyMetrix Research, 3862 Mexico Road, St. Peters, MO 63303, USA

Full list of author information is available at the end of the article
}

C C The Author(s). 2021 Open Access This article is licensed under a Creative Commons Attribution 4.0 International License, which permits use, sharing, adaptation, distribution and reproduction in any medium or format, as long as you give appropriate credit to the original author(s) and the source, provide a link to the Creative Commons licence, and indicate if changes were made. The images or other third party material in this article are included in the article's Creative Commons licence, unless indicated otherwise in a credit line to the material. If material is not included in the article's Creative Commons licence and your intended use is not permitted by statutory regulation or exceeds the permitted use, you will need to obtain permission directly from the copyright holder. To view a copy of this licence, visit http://creativecommons.org/licenses/by/4.0/ The Creative Commons Public Domain Dedication waiver (http://creativecommons.org/publicdomain/zero/1.0/) applies to the data made available in this article, unless otherwise stated in a credit line to the data. 


\section{Background}

Affecting more than 1 billion people worldwide, migraine is a major cause of disability, especially in young adult and middle-aged women [1, 2]. Patients suffering from migraine, especially those experiencing migraine with aura, have an increased relative risk for vascular comorbidities and events, such as stroke and myocardial ischemia, as compared to patients without migraine (or migraine without aura) [3-7]. Given the high frequency of both migraine and cardiovascular risk factors in the general population, it is important to assess the coexistence of these two risk factors and the potential impact of long-term migraine treatment.

Calcitonin gene-related peptide (CGRP) is currently thought to be central in migraine headache pathophysiology $[8,9]$ and is known to be a microvascular vasodilator [10]. There are currently four monoclonal antibodies approved by the U.S. Food and Drug Administration (FDA) for the prevention of migraine that either bind to the CGRP ligand (fremanezumab-vfrm; galcanezumabglnm; and eptinezumab-jjmr) or to the canonical CGRP receptor (erenumab-aooe [11]). Three have also been approved by the European Medicines Agency (fremanezumab-vfrm; galcanezumab-glnm; and erenumab-aooe). In addition, small-molecule CGRP receptor antagonists are approved by the FDA for acute treatment of migraine (rimegepant [12] and ubrogepant [13]) and migraine prevention (rimegepant [12] and atogepant [14]). Because CGRP mediates vasodilation [10], any therapy that inhibits the CGRP pathway may antagonize compensatory vasodilation. A study in isolated rat hearts found that CGRP is able to attenuate myocardial ischemia induced by a vasoconstrictor peptide [15]. Recently, a study in mice found that gepants (olcegepant and rimegepant), which are CGRP-receptor antagonists, worsened ischemic stroke [16], raising concerns that CGRP antagonism may increase the potential risk in patients at-risk for stroke or myocardial infarction. However, the overall importance of the CGRP pathway, when compared with other vasodilatory pathways, during ischemia (e.g., myocardial ischemia) is yet to be established $[6,10]$.

Acute migraine-specific medications (such as triptans and ergots) may rarely cause clinically meaningful vasoconstriction [17], and evidence of significant cardiovascular risk with triptans is lacking. Post-marketing data for erenumab, a CGRP-receptor antibody indicated for the preventive treatment of migraine in adults, suggest development or worsening of hypertension, leading to inclusion under Warnings and Precautions in its prescribing information [11]. However, results from a longterm, open-label extension study found erenumab to be safe in populations at cardiovascular risk [18]. Postmarket surveillance of CGRP-targeting monoclonal antibodies, galcanezumab [19] and fremanezumab [20], indicate no significant development or worsening hypertension when compared to placebo. As such, it is important to evaluate and understand the cardiovascular safety of all therapies that block the effects of CGRP in patients with migraine, particularly over the long term.

Eptinezumab (eptinezumab-jimr; ALD403), a humanized immunoglobulin G1 (IgG1) monoclonal antibody that binds the CGRP ligand with high affinity [21], is approved in the United States and other countries for the preventive treatment of migraine in adults. Four clinical trials found eptinezumab administered via 30-min intravenous infusion every 12 weeks to be safe, well tolerated, and effective in the prevention of episodic and chronic migraine [22-25]. These trials evaluated a range of eptinezumab doses, including $10 \mathrm{mg}, 30 \mathrm{mg}, 100 \mathrm{mg}, 300 \mathrm{mg}$, and $1000 \mathrm{mg}$, and included a phase $1 \mathrm{~b}$ trial in patients with episodic migraine (EM), a phase 2 trial in patients with chronic migraine $(\mathrm{CM})$, and the two pivotal phase 3 trials, PROMISE-1 in patients with EM and PROMISE-2 in patients with CM. PROMISE-1 and PROMISE-2 found that eptinezumab $100 \mathrm{mg}$ and $300 \mathrm{mg}$ met the primary efficacy endpoint of significantly reducing mean monthly migraine headache days over weeks 1 12 [24, 25], with preventive benefits observed in the $24 \mathrm{~h}$ after dosing [26]. Based on these findings, the approved doses in the United States are eptinezumab $100 \mathrm{mg}$ and $300 \mathrm{mg}$ administered intravenously for the prevention of migraine. In addition, the follow-up PREVAIL trial found that improvements in patientreported outcomes were generally sustained through week 104 [27].

To determine the effect of eptinezumab on cardiovascular co-morbidities and events in patients with migraine, the present analysis pooled the patient-level safety data from the placebo-controlled clinical trial program for eptinezumab in migraine prevention, focusing specifically on the approved 100-mg and 300-mg eptinezumab doses. This analysis also included the unapproved high dose of $1000 \mathrm{mg}$, which is over 3 times the maximum approved dose for eptinezumab. To evaluate the effect of eptinezumab treatment on cardiovascular comorbidities and events in patients with $\mathrm{EM}$ or $\mathrm{CM}$, this analysis determined if the rate of cardiovascular adverse events (AEs) was different for patients treated with eptinezumab, compared with patients receiving placebo. A secondary analysis was conducted to investigate the effects of treatment on blood pressure.

\section{Methods}

\section{Design}

Detailed methodology for four trials in the eptinezumab clinical trial program have been published previously [22-25]. In addition, the integrated safety analysis of all 
human studies involved in the clinical development has also been published [28]. The studies included in this pooled analysis are detailed in Table 1 . The PREVAIL study, which was an open-label study in which patients were treated with eptinezumab $300 \mathrm{mg}$ for up to 2 years, was not included in this integrated analysis due to the lack of a placebo arm [27].

\section{Reporting}

Study protocols and safety reporting documents for individual trials were reviewed in each of the regions where the studies were conducted, with each phase 3 trial (PROMISE-1, PROMISE-2) having an active Data and Safety Monitoring Board. Study documents, including data collection methods, were reviewed and approved at a local investigator site level before study initiation, with each site approved for appropriateness and ability to complete the required documentation. During study conduct, all information related to AEs was either spontaneously reported by the patient or in response to the investigator's non-directed questioning regarding safety, per the standard procedures recommended by the FDA and outlined in the clinical study protocols. For the purposes of this analysis, any reported AEs and all laboratory data (including blood pressure results) for the four placebo-controlled migraine prevention studies were then integrated and pooled.

\section{Eligibility criteria}

All four studies had similar inclusion/exclusion criteria, with each study having a screening period of 4 weeks, followed by a 4-week baseline period to confirm study eligibility before the patient was randomized. Key inclusion criteria at screening were age 18 years or older and a history of migraine with or without aura for at least 12 months. The CM studies included patients who experienced at least 15 headache days per month during the baseline period, of which at least 8 were documented migraine days. The EM studies included patients who reported between 4 and 14 headache days per month during the baseline period, of which at least 4 were reported as migraine days.
In the phase 3, PROMISE-1 EM study, the use of medications for acute headache before screening was restricted to acute migraine-specific medications (triptan or ergots; limited to $\leq 10$ days per month), analgesics or nonsteroidal anti-inflammatory drugs (NSAIDs; limited to $\leq 14$ days per month), and opioid-/butalbital-containing medications (limited to $\leq 4$ days per month). In the phase 3 PROMISE-2 CM study, there was no restriction on the acute migraine-specific medications, analgesics, or NSAIDs during the screening period, with a total of 431 patients $(40.2 \%)$ diagnosed with medication-overuse headache at baseline. Patients could not, however, have used opioid- or butalbital-containing medications on $>4$ days per month during the 3 months prior to enrollment. In the phase 3 migraine prevention studies, after randomization had been completed, patients could use acute migraine-specific medications, analgesics, NSAIDs, or other medications as needed to treat acute migraine.

In the phase $1 \mathrm{~b}$, phase 2 , and PROMISE-1 studies, patients were excluded due to pre-existing cardiovascular disease (hypertension, ischemic heart diseases), neurological disease, cerebrovascular disease, diabetes, or Raynaud's disease. In PROMISE-2, patients with active, progressive, or unstable cardiovascular disorders were excluded. In situations that were unclear, the investigator was to contact the medical monitor for guidance. Patients were excluded from the migraine prevention studies if they had a history or evidence of atherosclerosis, cardiomyopathy, coronary artery disease, diabetes, serious heart rhythm abnormalities, or other cardiovascular diseases. The studies for eptinezumab used in this post hoc analysis included patients with allowable cardiovascular risk factors including a medical history of hyperlipidemia-related conditions or conditions leading to higher likelihood of diabetes development; obesity (Class I, Class II, and Class III); an age range of 18 to 75 years (including male patients aged $\geq 45$ years, and female patients aged $\geq 55$ years); and race (including patients who reported as Black or African American) [29]. Patients with a medical history of hyperlipidemia-related conditions included dyslipidemia, hypercholesterolemia, hyperlipidemia, hypertriglyceridemia, or lipid

Table 1 Eptinezumab Clinical Studies

\begin{tabular}{|c|c|c|c|c|}
\hline Study & Description & Migraine Diagnosis & Dosing Frequency & Follow-up Visits \\
\hline NCT01772524 [22] & $\begin{array}{l}\text { Phase 1b } \\
D B / P C / P G / R\end{array}$ & EM & $\begin{array}{l}1000 \text { mg, placebo } \\
\text { Single dose (day 0) }\end{array}$ & Weeks $4,8,12,24$ \\
\hline NCT02275117 [23] & $\begin{array}{l}\text { Phase } 2 \\
\mathrm{DB} / \mathrm{PC} / \mathrm{PG} / \mathrm{R}\end{array}$ & CM & $\begin{array}{l}10,30,100,300 \mathrm{mg} \text {, placebo } \\
\text { Single dose (day 0) }\end{array}$ & Weeks $4,8,12,24,36,49$ \\
\hline PROMISE-1 (NCT02559895) [24] & $\begin{array}{l}\text { Phase } 3 \\
\mathrm{DB} / \mathrm{PC} / \mathrm{PG} / \mathrm{R}\end{array}$ & EM & $\begin{array}{l}30,100,300 \mathrm{mg} \text {, placebo } \\
\text { Four doses (day 0, weeks 12, 24, 36) }\end{array}$ & Weeks $4,8,12,16,20,24,28,36,48,56$ \\
\hline PROMISE-2 (NCT02974153) [25] & $\begin{array}{l}\text { Phase } 3 \\
\mathrm{DB} / \mathrm{PC} / \mathrm{PG} / \mathrm{R}\end{array}$ & CM & $\begin{array}{l}\text { 100, } 300 \text { mg, placebo } \\
\text { Two doses (day 0, week 12) }\end{array}$ & Weeks $2,4,8,12,16,20,24,32$ \\
\hline
\end{tabular}

$D B$ Double-blind, $P C$ Placebo-controlled, $P G$ Parallel-group, $R$ Randomized 
metabolism disorder; conditions leading to higher likelihood of diabetes development included glucose tolerance impaired, hyperglycemia, or impaired fasting glucose.

\section{Outcome measures}

In all studies, AEs that emerged after the first dose of study treatment (eptinezumab $100 \mathrm{mg}, 300 \mathrm{mg}, 1000 \mathrm{mg}$, or placebo) were recorded through study completion. The Medical Dictionary for Regulatory Activities (MedDRA) was used in all of the studies (Version 15.0 in phase 1 and 2 trials and version 20.1 in phase 3 trials) to determine the preferred term and system organ class for each $\mathrm{AE}$, with severity graded according to Common Terminology Criteria for Adverse Events (CTCAE) criteria, version 4.03. Per the CTCAE criteria, a serious AE was defined as having met at least 1 of the following criteria: fatal, life-threatening, requiring inpatient hospitalization or prolongation of existing hospitalization, resulting in persistent or significant disability/incapacity, congenital anomaly/birth defect, or another medically important event. During the phase 3 clinical trials, a Data Monitoring Committee adjudicated each potential cardiovascular treatment-emergent adverse event (TEAE) based on pre-specified definitions, including death, acute myocardial infarction or hospitalization for unstable angina event, non-fatal stroke or transient ischemic attack, coronary revascularization procedure, hospitalization for hypertension, hospitalization for peripheral artery disease, or revascularization procedure for peripheral artery disease. Study drug discontinuation and infusion interruption due to cardiovascular TEAEs was monitored, as were any new or changed cardiovascular concomitant medications.

In all studies, vital signs measurements, including resting blood pressure and heart rate, were captured at the baseline visit, each 12-week study visit for dosing, and the study conclusion visit. In PROMISE-2, measurements were captured prior to dosing and at $2 \mathrm{~h}( \pm 30$ min) after administration end; in all other studies, measurements were captured prior to dosing and at $4 \mathrm{~h}( \pm$ $30 \mathrm{~min}$ ) after administration end.

\section{Statistical analysis}

Integrated analyses of AEs were conducted by assigned treatment (eptinezumab $100 \mathrm{mg}, 300 \mathrm{mg}, 1000 \mathrm{mg}$, or placebo), with patients summarized within the treatment group that they received. For patients treated with two different dose levels, they were summarized in the treatment arm of the highest dose level received. No patient who received eptinezumab $1000 \mathrm{mg}$ received any other dose level because the phase 1 (NCT01772524) was single-dose and because patients in the phase 1 and phase 2 (NCT02275117) studies were excluded from the phase 3 (NCT02559895 or NCT02974153) studies. To analyze for specific cardiovascular AEs, standardized MedDRA queries with narrow search terms were used to determine the overall incidences in each treatment group in the following categories: ischemic central nervous system vascular conditions, ischemic heart disease, peripheral artery disease, and hypertension. The integrated pooled population was utilized to summarize resting systolic blood pressure (BP), diastolic $\mathrm{BP}$, and pulse rate at each study visit, with mean values ( \pm standard deviation $[\mathrm{SD}]$ ) calculated.

\section{Results}

The main results of the four trials have been published previously [22-25] and, in general, eptinezumab was found to be safe, well-tolerated, and efficacious in preventing migraine. Demographics and baseline characteristics for the individual clinical trials have been reported [22-25] and were similar among treatment and placebo groups. Baseline demographics and characteristics of the pooled population are presented in Table 2 , with the groups being well matched across baseline characteristics. A total of $N=2268$ patients with migraine were included in this analysis, including patients receiving eptinezumab $100 \mathrm{mg}, n=701$; eptinezumab $300 \mathrm{mg}, n=$ 695; eptinezumab $1000 \mathrm{mg}, n=81$; placebo, $n=791$. Regarding cardiovascular risk factors, $31.3 \%$ of patients were obese (Class I or II), and about half of the population was $\geq 40$ years. Approximately $48 \%$ and $13 \%$ of the patient population had $\geq 1$ and $\geq 2$ cardiovascular risk factors, respectively. Although the individual trials varied in number of doses administered and overall treatment duration, more than $97 \%$ of patients had study drug exposure for $\geq 12$ weeks (see Table 3 ).

\section{Cardiovascular treatment-emergent adverse events}

The pooled patient-level analysis of cardiovascular TEAEs is presented in Table 4. In general, there was a limited incidence of cardiovascular TEAEs across all four clinical trials, with rates similar between the eptinezumab groups and placebo. Cardiovascular TEAEs were mild or moderate in severity (grades 1 and 2, respectively), with none graded $3-5$ or as serious or lifethreatening AEs. The evaluation of cardiovascular TEAEs stratified by patients with $\geq 1$ and $\geq 2$ cardiovascular risk factors at baseline are presented in Table 5 . Although cardiovascular TEAEs were limited and not severe in nature, the majority occurred in patients with $\geq 1$ and $\geq 2$ cardiovascular risk factors at baseline and no differences in incidence were reported between the eptinezumab- and placebo-treated patient groups. Also, there was no signal of any dose-related incidence in the eptinezumab 100-mg, 300-mg, or 1000-mg treatment arms. 
Table 2 Baseline Demographics and Characteristics

\begin{tabular}{|c|c|c|c|c|}
\hline & $\begin{array}{l}\text { Eptinezumab } 100 \mathrm{mg} \\
N=701\end{array}$ & $\begin{array}{l}\text { Eptinezumab } 300 \mathrm{mg} \\
\mathrm{N}=695\end{array}$ & $\begin{array}{l}\text { Eptinezumab } 1000 \mathrm{mg} \\
\mathrm{N}=81\end{array}$ & $\begin{array}{l}\text { Placebo } \\
N=791\end{array}$ \\
\hline Mean age, years (SD) & $39.9(11.11)$ & $40.1(10.84)$ & $38.6(10.81)$ & $39.3(10.96)$ \\
\hline Sex: Female, n (\%) & $590(84.2)$ & $611(87.9)$ & $67(82.7)$ & $686(86.7)$ \\
\hline \multicolumn{5}{|l|}{ Race, n (\%) } \\
\hline White & $636(90.7)$ & $623(89.6)$ & $66(81.5)$ & $677(85.6)$ \\
\hline Black / African American & $50(7.1)$ & $54(7.8)$ & $10(12.3)$ & $84(10.6)$ \\
\hline Asian & $2(0.3)$ & $3(0.4)$ & $4(4.9)$ & $7(0.9)$ \\
\hline American Indian / Alaska Native & $1(0.1)$ & $3(0.4)$ & 0 & $3(0.4)$ \\
\hline Native Hawaiian / other Pacific Islander & $1(0.1)$ & $2(0.3)$ & 0 & $2(0.3)$ \\
\hline Multiple races & $9(1.3)$ & $8(1.2)$ & $1(1.2)$ & $13(1.6)$ \\
\hline Other & $2(0.3)$ & $1(0.1)$ & 0 & $5(0.6)$ \\
\hline Not reported & 0 & $1(0.1)$ & 0 & 0 \\
\hline Mean $\mathrm{BMl}, \mathrm{kg} / \mathrm{m}^{2}$ & $27.6(6.15)$ & $27.3(5.91)$ & $27.5(5.17)$ & $27.9(6.14)$ \\
\hline Preventive migraine medication use, $\mathrm{n}(\%)^{*}$ & $52 / 478(10.9)$ & $62 / 471(13.2)$ & - & $49 / 487(10.1)$ \\
\hline \multicolumn{5}{|l|}{ Cardiovascular risk factors, n (\%) } \\
\hline Hypertension-related & $36(5.1)$ & $26(3.7)$ & $1(1.2)$ & $28(3.5)$ \\
\hline Hyperlipidemia-related & $48(6.8)$ & $45(6.5)$ & $5(6.2)$ & $45(5.7)$ \\
\hline Diabetes-related & $3(0.4)$ & 0 & $1(1.2)$ & $5(0.6)$ \\
\hline History of ischemic CV events/procedures & $4(0.6)$ & $3(0.4)$ & 0 & $3(0.4)$ \\
\hline Obesity (Class 1 or 2), BMl $\geq 30 \mathrm{~kg} / \mathrm{m}^{2}$ & $226(32.2)$ & $197(28.3)$ & $26(32.1)$ & $265(33.5)$ \\
\hline Male $\geq 45$ years & $43(6.1)$ & $24(3.5)$ & $4(4.9)$ & $38(4.8)$ \\
\hline Female $\geq 55$ years & $63(9.0)$ & $57(8.2)$ & $2(2.5)$ & $49(6.2)$ \\
\hline Black or African American race & $50(7.1)$ & $54(7.8)$ & $10(12.3)$ & $84(10.6)$ \\
\hline$\geq 1 \mathrm{CV}$ risk factor & $350(49.9)$ & $316(45.5)$ & $41(50.6)$ & $382(48.3)$ \\
\hline$\geq 2 \mathrm{CV}$ risk factors & $97(13.8)$ & $83(11.9)$ & $7(8.6)$ & $112(14.2)$ \\
\hline \multicolumn{5}{|l|}{ Medical/Surgical CV history, n (\%) } \\
\hline Angina pectoris & 0 & $1(0.1)$ & 0 & 0 \\
\hline Aortic valve incompetence & $1(0.1)$ & 0 & 0 & 0 \\
\hline Arrhythmia & 0 & 0 & $1(1.2)$ & $1(0.1)$ \\
\hline Supraventricular arrhythmia & 0 & $1(0.1)$ & 0 & 0 \\
\hline Atrioventricular block, first degree & 0 & 0 & 0 & $1(0.1)$ \\
\hline Bradycardia & $2(0.3)$ & $4(0.6)$ & 0 & $4(0.5)$ \\
\hline Bundle branch block, left & $1(0.1)$ & 0 & 0 & 0 \\
\hline Bundle branch block, right & 0 & $2(0.3)$ & 0 & $2(0.3)$ \\
\hline Cardiomegaly & $1(0.1)$ & 0 & 0 & 0 \\
\hline Cardiomyopathy & 0 & $1(0.1)$ & 0 & 0 \\
\hline Mitral valve incompetence & $1(0.1)$ & 0 & 0 & $1(0.1)$ \\
\hline Mitral valve prolapse & $1(0.1)$ & $4(0.6)$ & 0 & $6(0.8)$ \\
\hline Palpitations & $3(0.4)$ & $2(0.3)$ & 0 & $5(0.6)$ \\
\hline POTS & $1(0.1)$ & 0 & 0 & 0 \\
\hline Sinus arrhythmia & $1(0.1)$ & $1(0.1)$ & 0 & 0 \\
\hline Sinus bradycardia & 0 & 0 & 0 & $2(0.3)$ \\
\hline Sinus tachycardia & $1(0.1)$ & $1(0.1)$ & 0 & $1(0.1)$ \\
\hline Supraventricular tachycardia & $1(0.1)$ & $2(0.3)$ & 0 & $1(0.1)$ \\
\hline
\end{tabular}


Table 2 Baseline Demographics and Characteristics (Continued)

\begin{tabular}{lllll}
\hline & Eptinezumab $\mathbf{1 0 0} \mathbf{~ m g}$ & Eptinezumab $\mathbf{3 0 0} \mathbf{~ m g}$ & Eptinezumab 1000 mg & Placebo \\
& $\mathbf{N = 7 0 1}$ & $\mathbf{N = 6 9 5}$ & $\mathbf{N = 8 1}$ & $\mathbf{N = 7 9 1}$ \\
\hline Tachycardia & 0 & $2(0.3)$ & $1(1.2)$ & $5(0.6)$ \\
Tricuspid valve incompetence & $1(0.1)$ & 0 & 0 & 0 \\
Ventricular extrasystoles & $1(0.1)$ & 0 & 0 & $1(0.1)$ \\
Ventricular tachycardia & 0 & 0 & 0 & $1(0.1)$ \\
\hline
\end{tabular}

*Use of a stable preventive migraine medication regimen was permitted in CM studies, and as such, the number of patients reflects only those studies. BMI body mass index, CM Chronic migraine, CV Cardiovascular, POTS Postural orthostatic tachycardia syndrome, SD Standard deviation

In total, 27 cardiac or vascular TEAEs occurred that were deemed directly related to treatment. Of these, 8 occurred in patients treated with eptinezumab $100 \mathrm{mg}$ ( $n=1$ each for sinus tachycardia, increased BP, increased systolic BP, ECG ST segment depression, hypertension, and flushing; $n=2$ for hot flushes), 7 occurred in patients treated with eptinezumab $300 \mathrm{mg}$ ( $n=1$ each for atrioventricular first degree block, ECG T-wave inversion, increased heart rate; $n=2$ each for hot flushes and flushing), 6 occurred in patients treated with eptinezumab $1000 \mathrm{mg}$ ( $n=1$ each for ECG abnormal Q-wave, ECG abnormal T-wave, and hot flush; $n=3$ for ECG QT prolongation), and 6 occurred in patients treated with placebo ( $\mathrm{n}=1$ each for ECG abnormal T-wave, hypertension, flushing, and pre-hypertension; $n=2$ for palpitations).

The incidence of study drug discontinuations and infusion interruptions due to TEAEs are available in Supplemental Table 1. Across the pooled population comprising the four eptinezumab migraine prevention studies, a total of $9(1.3 \%)$ and $13(1.9 \%)$ patients in the eptinezumab 100-mg and eptinezumab 300-mg groups, respectively, and $8(1.0 \%)$ patients receiving placebo discontinued study drug due to TEAEs; no patient discontinued in the eptinezumab 1000-mg group due to TEAEs. Hypertension led to 1 (0.1\%) patient receiving $100 \mathrm{mg}$ eptinezumab discontinuing study drug use. However, no patients discontinued in the 300 - or 1000 -mg eptinezumab groups or in the placebo groups, and no other cardiovascular TEAEs resulted in treatment discontinuation. A total of 11 (1.6\%), $13(1.9 \%)$, and $6(0.8 \%)$ patients in the eptinezumab 100-mg, eptinezumab 300-mg, and placebo groups, respectively, had an infusion interruption due to TEAEs; none were cardiovascular in nature. No patients had infusion interruptions due to a TEAE in the eptinezumab 1000-mg group.

The assessment of vital signs over time in the pooled population is presented in Fig. 1, with the readings being similar across the treatment groups and across the study visits. Mean (SD) change from baseline to week 12 in systolic BP (see Fig. 1A) was -0.4 (10.2) $\mathrm{mm} \mathrm{Hg}$ in the eptinezumab 100-mg group, 0.2 (10.6) $\mathrm{mm} \mathrm{Hg}$ in the eptinezumab $300-\mathrm{mg}$ group, -1.0 (11.8) $\mathrm{mm} \mathrm{Hg}$ in the eptinezumab 1000$\mathrm{mg}$ group, and $0.5(10.5) \mathrm{mm} \mathrm{Hg}$ in the placebo group. Mean (SD) change from baseline to week 12 in diastolic BP (see Fig. 1B) was $0.4(8.2) \mathrm{mm} \mathrm{Hg}$ in the eptinezumab 100-mg group, $-0.3(7.9) \mathrm{mm} \mathrm{Hg}$ in the eptinezumab 300-mg group, 0.7 (7.9) $\mathrm{mm} \mathrm{Hg}$ in the eptinezumab 1000-mg group, and -0.1 (7.5) $\mathrm{mm}$ $\mathrm{Hg}$ in the placebo group. Similar results were observed at week $24(100 \mathrm{mg}, 300 \mathrm{mg}, 1000 \mathrm{mg}$, and placebo) as well as through week $56(100 \mathrm{mg}, 300 \mathrm{mg}$, and placebo only). There were no clinically relevant differences across treatment groups. Similar results were observed in changes in resting heart rate (see Fig. 1C), with a mean (SD) change from baseline to week 12 of 0.0 (9.7) beats per minute (bpm) in the eptinezumab 100-mg group, -0.2 (10.4) bpm in the

Table 3 Clinical Study Duration

\begin{tabular}{|c|c|c|c|c|}
\hline & $\begin{array}{l}\text { Eptinezumab } 100 \mathrm{mg} \\
N=701\end{array}$ & $\begin{array}{l}\text { Eptinezumab } 300 \mathrm{mg} \\
\mathrm{N}=695\end{array}$ & $\begin{array}{l}\text { Eptinezumab } 1000 \mathrm{mg} \\
\mathrm{N}=81\end{array}$ & $\begin{array}{l}\text { Placebo } \\
N=791\end{array}$ \\
\hline \multicolumn{5}{|c|}{ Total Time in Clinical Study, n (\%) } \\
\hline$\geq 12$ weeks & $680(97.0)$ & $681(98.0)$ & $78(96.3)$ & $766(96.8)$ \\
\hline$\geq 24$ weeks & $642(91.6)$ & $649(93.4)$ & $46(56.8)$ & $692(87.5)$ \\
\hline$\geq 36$ weeks & $288(41.1)$ & $293(42.2)$ & N/A & $280(35.4)$ \\
\hline$\geq 48$ weeks & $263(37.5)$ & $270(38.8)$ & N/A & $256(32.4)$ \\
\hline
\end{tabular}

Total Exposure Time $=$ Last day on study - first dose date +1. N/A Not applicable 
Table 4 Treatment-Emergent Cardiovascular Adverse Events

\begin{tabular}{|c|c|c|c|c|}
\hline & Eptinezumab $100 \mathrm{mg}$ & Eptinezumab 300 mg & Eptinezumab 1000 mg & Placebo \\
\hline Patients, n (\%) & $N=701$ & $N=695$ & $N=81$ & $N=791$ \\
\hline Cardiac disorders & $6(0.9)$ & $8(1.2)$ & $2(2.5)$ & $8(1.0)$ \\
\hline Bradycardia & 0 & $2(0.3)$ & 0 & 0 \\
\hline Palpitations & $1(0.1)$ & $2(0.3)$ & 0 & $3(0.4)$ \\
\hline Tachycardia & $1(0.1)$ & $2(0.3)$ & 0 & $1(0.1)$ \\
\hline Atrial fibrillation & 0 & $1(0.1)$ & 0 & $1(0.1)$ \\
\hline Atrioventricular block first degree & $2(0.3)$ & $1(0.1)$ & 0 & $1(0.1)$ \\
\hline Bundle branch block right & 0 & 0 & $1(1.2)$ & $1(0.1)$ \\
\hline Nodal rhythm & 0 & 0 & $1(1.2)$ & 0 \\
\hline Sinus bradycardia & 0 & 0 & 0 & $1(0.1)$ \\
\hline Sinus tachycardia & $1(0.1)$ & 0 & 0 & 0 \\
\hline Supraventricular extrasystoles & $1(0.1)$ & 0 & 0 & 0 \\
\hline Investigations $^{\mathrm{a}}$ & $37(5.3)$ & $28(4.0)$ & $10(12.3)$ & $39(4.9)$ \\
\hline Increased blood pressure & $7(1.0)$ & $3(0.4)$ & $1(1.2)$ & $5(0.6)$ \\
\hline Increased heart rate & 0 & $1(0.1)$ & 0 & 0 \\
\hline Increased systolic blood pressure & $1(0.1)$ & 0 & 0 & 0 \\
\hline Abnormal electrocardiogram Q wave & $1(0.1)$ & 0 & $1(1.2)$ & 0 \\
\hline Prolonged electrocardiogram QT & 0 & 0 & $3(3.7)$ & $1(0.1)$ \\
\hline Vascular disorders & $11(1.6)$ & $10(1.4)$ & $1(1.2)$ & $8(1.0)$ \\
\hline Hot flush & $2(0.3)$ & $4(0.6)$ & $1(1.2)$ & 0 \\
\hline Hypertension & $4(0.6)$ & $3(0.4)$ & 0 & $6(0.8)$ \\
\hline Flushing & $1(0.1)$ & $2(0.3)$ & 0 & $1(0.1)$ \\
\hline Hypotension & $1(0.1)$ & $1(0.1)$ & 0 & 0 \\
\hline Prehypertension & 0 & 0 & 0 & $1(0.1)$ \\
\hline
\end{tabular}

${ }^{\mathrm{a}}$ Not limited to CV-related investigations. CV Cardiovascular

eptinezumab 300-mg group, -0.5 (9.8) bpm in the eptinezumab 1000-mg group, and 0.4 (9.8) bpm in the placebo group. Similar results were observed at week 24 and week 56, with no clinically relevant differences across treatment groups.

The analysis of new or changed concomitant cardiovascular medications is presented in Supplemental Table 2. In general, treatment with eptinezumab (any dose) did not lead to new or changed cardiovascularrelated concomitant medications, with the overall rates similar to placebo.

\section{Discussion}

Across the four clinical studies, the incidences of AEs were similar across eptinezumab and placebo treatment groups, with no evidence of dose dependency for eptinezumab with regard to cardiovascular safety outcomes. The review of both patient-level and aggregate AEs did not find evidence of an association between eptinezumab treatment and any cardiovascular event. Based on the low number of cardiovascular events that occurred in any treatment group, it was not possible to conduct any further subgroup analysis, such as in patients with acute migraine-specific medication use at baseline. There was no evidence that patients treated with eptinezumab who used acute migraine-specific medications had any greater incidence of serious adverse events compared with nonusers of acute migraine-specific medications. Because CGRP contributes to the maintenance and regulation of cardiovascular homeostasis, these results are of clinical relevance for patients with migraine and health care providers [30].

Among patients with at least one cardiovascular risk factor at baseline, the incidence of AEs was similar across the eptinezumab and placebo treatment groups and independent of the presence (or absence) of cardiovascular risk factors at baseline. Similarly, eptinezumab treatment for up to 56 weeks had no relevant effect on blood pressure or heart rate, with no differences across treatment groups, including in patients who received eptinezumab $1000 \mathrm{mg}$. Collectively, these 
Table 5 Treatment-Emergent Cardiovascular Adverse Events in Patients with Cardiovascular Risk Factors at Baseline

\begin{tabular}{|c|c|c|c|c|}
\hline Patients with at least 1 cardiovascular risk factor, $n$ (\%) & $\begin{array}{l}\text { Eptinezumab } 100 \mathrm{mg} \\
N=350\end{array}$ & $\begin{array}{l}\text { Eptinezumab } 300 \mathrm{mg} \\
N=316\end{array}$ & $\begin{array}{l}\text { Eptinezumab } 1000 \mathrm{mg} \\
N=41\end{array}$ & $\begin{array}{l}\text { Placebo } \\
N=382\end{array}$ \\
\hline Any TEAE & $196(56.0)$ & $187(59.2)$ & $22(53.7)$ & $220(57.6)$ \\
\hline Cardiac or Vascular TEAEs & $13(3.7)$ & $14(4.4)$ & $4(9.8)$ & $19(5.0)$ \\
\hline Increased blood pressure & $5(1.4)$ & $3(0.9)$ & 0 & $2(0.5)$ \\
\hline Hypertension & $2(0.6)$ & $1(0.3)$ & 0 & $5(1.3)$ \\
\hline Hot flush & 0 & $3(0.9)$ & 0 & 0 \\
\hline Palpitations & $1(0.3)$ & $1(0.3)$ & 0 & $3(0.8)$ \\
\hline Syncope & $2(0.6)$ & $2(0.6)$ & 0 & $3(0.8)$ \\
\hline Tachycardia & 0 & $2(0.6)$ & 0 & $1(0.3)$ \\
\hline Chest pain & 0 & 0 & $1(2.4)$ & $1(0.3)$ \\
\hline ECG QT prolongation & 0 & 0 & $2(4.9)$ & $1(0.3)$ \\
\hline Flushing & 0 & $1(0.3)$ & 0 & $1(0.3)$ \\
\hline Sinus tachycardia & $1(0.3)$ & 0 & 0 & 0 \\
\hline Increased systolic blood pressure & $1(0.3)$ & 0 & 0 & 0 \\
\hline Abnormal ECG Q wave & 0 & 0 & $1(2.4)$ & 0 \\
\hline Increased heart rate & 0 & $1(0.3)$ & 0 & 0 \\
\hline Hypotension & $1(0.3)$ & 0 & 0 & 0 \\
\hline Atrial fibrillation & 0 & 0 & 0 & $1(0.3)$ \\
\hline Prehypertension & 0 & 0 & 0 & $1(0.3)$ \\
\hline Patients with at least 2 cardiovascular risk factors, $\mathbf{n}(\%)$ & $N=97$ & $N=83$ & $N=7$ & $N=112$ \\
\hline Any TEAE & $54(55.7)$ & $51(61.4)$ & $4(57.1)$ & $69(61.6)$ \\
\hline Cardiac or Vascular TEAEs & $8(8.2)$ & $4(4.8)$ & 0 & $8(7.1)$ \\
\hline Increased blood pressure & $2(2.1)$ & $1(1.2)$ & 0 & $1(0.9)$ \\
\hline Hypertension & $2(2.1)$ & $1(1.2)$ & 0 & $4(3.6)$ \\
\hline Syncope & $2(2.1)$ & $1(1.2)$ & 0 & $2(1.8)$ \\
\hline Palpitations & $1(1.0)$ & 0 & 0 & 0 \\
\hline Hot flush & 0 & $1(1.2)$ & 0 & 0 \\
\hline Sinus tachycardia & $1(1.0)$ & 0 & 0 & 0 \\
\hline Atrial fibrillation & 0 & 0 & 0 & $1(0.9)$ \\
\hline
\end{tabular}

ECG Electrocardiogram, TEAE Treatment-emergent adverse event

pooled, patient-level analyses support the cardiovascular safety profile of eptinezumab in the prevention of migraine across different study periods (up to 56 weeks), patient populations (EM and CM), and methodologies (single and multiple IV infusions), providing a comprehensive evaluation of the overall safety of eptinezumab for prevention of migraine.

\section{Limitations}

This analysis of cardiovascular safety utilized patientlevel data that were derived as part of the controlled setting of clinical trials. To generalize these data, additional evidence from real-world analyses would be needed to confirm the cardiovascular safety of eptinezumab in all patient populations. In addition, cardiovascular safety was not analyzed specifically in patients with migraine with aura, a known associate of cardiovascular comorbidities; however, a separate analysis found no increased risk for cardiovascular TEAEs in these patients [31]. Further, the studies used in this analysis excluded patients with evidence of significant cardiovascular disease (including those with uncontrolled or newly diagnosed hypertension) and diabetes; therefore, the long-term safety in high-risk patients remains unknown. Another limitation to this analysis is that tobacco use was not documented. While triptans and ergots have been associated with a higher risk of cardiovascular TEAEs [17, $32,33]$, we did not examine specific use of these acute migraine-specific medications in our patients due to small subgroup sizes. 
A. Eptinezumab $100 \mathrm{mg} \Vdash$ Eptinezumab $300 \mathrm{mg} \triangle$ Eptinezumab $1000 \mathrm{mg} \backsim$ Placebo

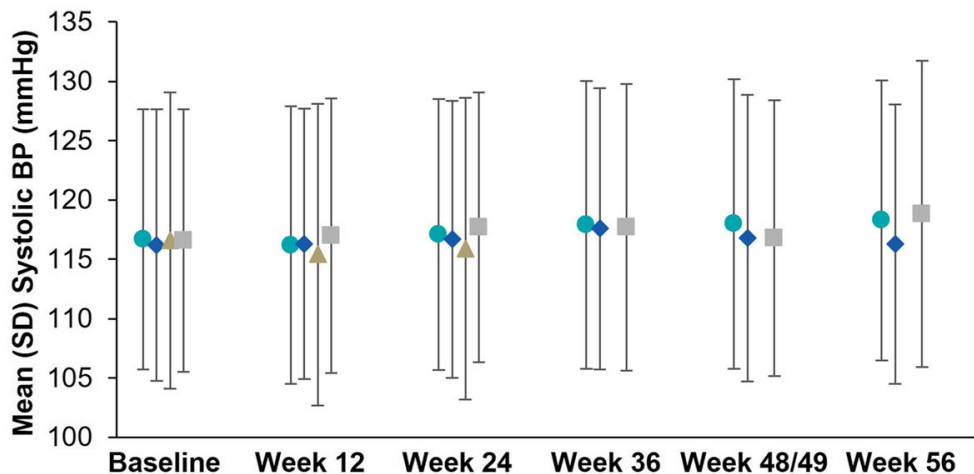

Sample sizes

$\begin{array}{rccccc}100 \mathrm{mg} 701 & 659 & 618 & 269 & 258 & 173 \\ 300 \mathrm{mg} 695 & 662 & 623 & 271 & 260 & 169 \\ 1000 \mathrm{mg} 81 & 76 & 74 & \text { NA } & \text { NA } & \text { NA } \\ \text { Placebo } 791 & 732 & 684 & 262 & 247 & 165\end{array}$

B. $\bullet$ Eptinezumab $100 \mathrm{mg} \diamond$ Eptinezumab $300 \mathrm{mg} \triangle$ Eptinezumab $1000 \mathrm{mg} \backsim$ Placebo
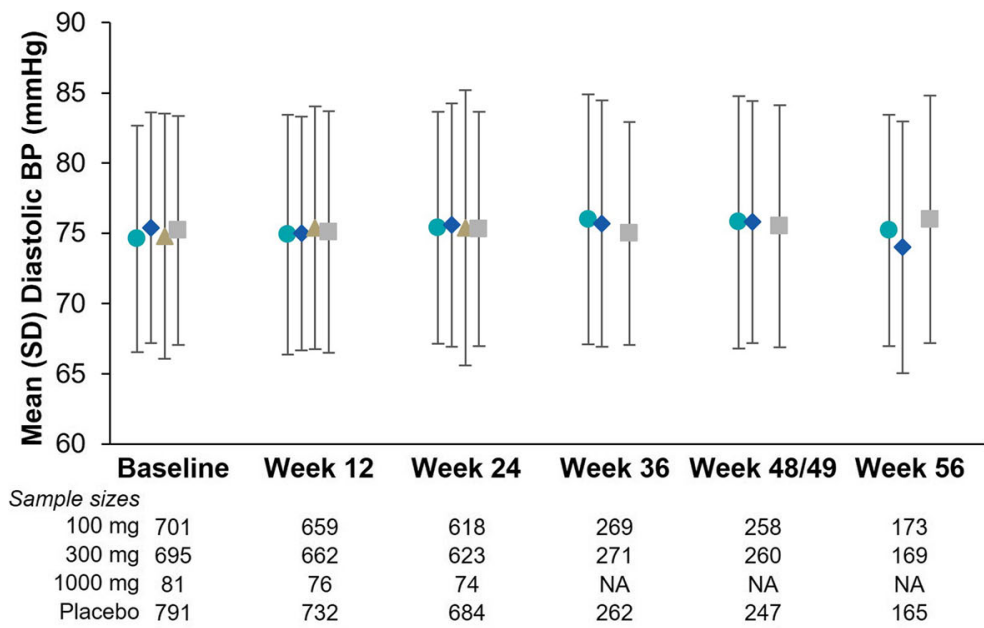

C. Eptinezumab $100 \mathrm{mg} \bowtie$ Eptinezumab $300 \mathrm{mg} \triangle$ Eptinezumab $1000 \mathrm{mg} \backsim$ Placebo
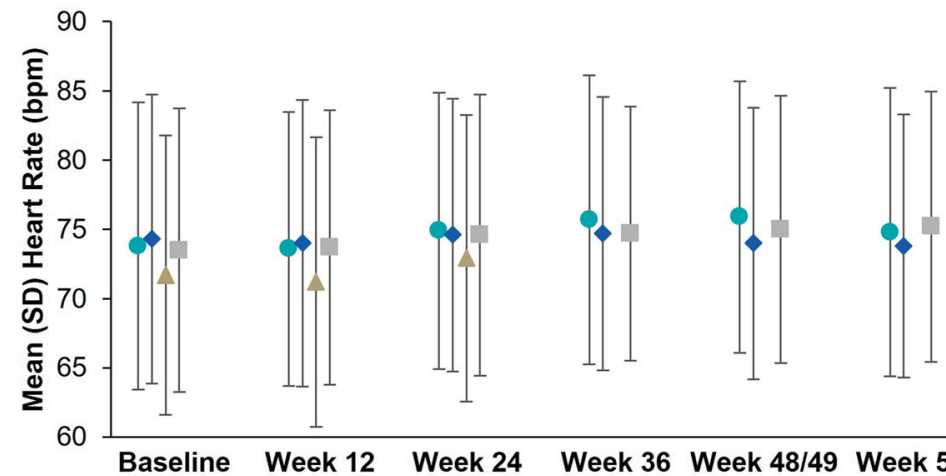

Sample sizes
$100 \mathrm{mg} 701$
$300 \mathrm{mg} 695$

$1000 \mathrm{mg} 81$

Placebo 791

659
662
76
732

618
623
74
684

269
271
NA
262

$\begin{array}{ll}258 & 173 \\ 260 & 169 \\ \text { NA } & \text { NA } \\ 247 & 165\end{array}$

Fig. 1 Mean Vital Sign Parameters Over Time: (A) Systolic Blood Pressure (BP), (B) Diastolic BP, and (C) Heart Rate 


\section{Conclusions}

This pooled, patient-level analysis of four clinical trials in patients with EM or CM found that eptinezumab at doses of $100 \mathrm{mg}, 300 \mathrm{mg}$, and $1000 \mathrm{mg}$ (which is more than 3 times the highest maximum approved dose) did not result in meaningful changes in blood pressure, heart rate, or concomitant cardiovascular-medication usage, and had comparable incidence of cardiovascular TEAEs to placebo. The overall incidence of cardiovascular TEAEs was low.

\section{Abbreviations}

AE: Adverse event; BP: Blood pressure; CV: Cardiovascular; CGRP: Calcitonin gene-related peptide; CM: Chronic migraine; CTCAE: Common terminology criteria for adverse events; EM: Episodic migraine; IgG1: Immunoglobulin G1; MedDRA: Medical Dictionary for Regulatory Activities; NSAID: Nonsteroidal anti-inflammatory drug; SD: Standard deviation; TEAE: Treatment-emergent adverse event

\section{Supplementary Information}

The online version contains supplementary material available at https://doi. org/10.1186/s10194-021-01360-1.

Additional file 1: Supplemental Table 1. Study Drug Discontinuation and Infusion Interruption Due to Cardiovascular Treatment-emergent Adverse Events Across the Pooled Eptinezumab Clinical Trial Population. Supplemental Table 2. New or Changed Cardiovascular Concomitant Medications Across the Pooled Eptinezumab Clinical Trial Population.

\section{Acknowledgements}

The authors thank Payal Thakkar, PhD, and Nicole Coolbaugh, CMPP, of The Medicine Group, LLC (New Hope, PA, United States) for providing medical writing support, which was funded by $\mathrm{H}$. Lundbeck A/S (Copenhagen, Denmark) in accordance with Good Publication Practice guidelines.

\section{Authors' contributions}

TRS, ELHS, RC, AE, and VS contributed to the conception and design of the study or data acquisition. JH performed the statistical analyses, and all authors contributed to interpretation of the data. All authors reviewed and provided critical revision of all manuscript drafts for important intellectual content, as well as read and approved the final manuscript for submission.

\section{Funding}

Sponsored and funded by $\mathrm{H}$. Lundbeck A/S. All authors and $\mathrm{H}$. Lundbeck $\mathrm{A} / \mathrm{S}$ and Lundbeck Seattle BioPharmaceuticals, Inc. prepared, reviewed, and approved the manuscript and made the decision to submit the manuscript for publication. Editorial support for the development of this manuscript was funded by $H$. Lundbeck A/S.

\section{Availability of data and materials}

In accordance with EFPIA's and PhRMA's "Principles for Responsible Clinical Trial Data Sharing" guidelines, Lundbeck is committed to responsible sharing of clinical trial data in a manner that is consistent with safeguarding the privacy of patients, respecting the integrity of national regulatory systems, and protecting the intellectual property of the sponsor. The protection of intellectual property ensures continued research and innovation in the pharmaceutical industry. Deidentified data are available to those whose request has been reviewed and approved through an application submitted to https://www.lundbeck.com/trials.

\section{Declarations}

\section{Ethics approval and consent to participate}

Each study included in this analysis was approved by an institutional review board or independent ethics committee. The studies were registered at ClinicalTrials.gov under NCT01772524, NCT02275117, NCT02559895, and
NCT02974153. In each study, patients provided written informed consent to participate.

\section{Consent for publication}

Not applicable.

\section{Competing interests}

TRS has been a consultant and/or scientific advisor for Alder/Lundbeck, Amgen, Biohaven, Eli Lilly, Impel Neuropharma, and Theranica, and has received research support from Alder/Lundbeck, Allergan, Amgen, Biohaven, Charleston Labs, Eli Lilly, electroCore, Novartis, Novo Nordisk, Satsuma, Theranica, and Vorso.

ELHS received research grants as a clinical trial investigator from Alder BioPharmaceuticals and is also a member of Lundbeck's Speaker Bureau. $\mathrm{RC}, \mathrm{AE}$, and VS are employees of Lundbeck or one of its subsidiary companies.

$\mathrm{JH}$ is an employee of Pacific Northwest Statistical Consulting, Inc., a contracted service provider of biostatistical resources for $\mathrm{H}$. Lundbeck A/S.

\section{Author details}

1StudyMetrix Research, 3862 Mexico Road, St. Peters, MO 63303, USA. ${ }^{2}$ Medvadis Research Corporation, Boston PainCare, Waltham, MA, USA. ${ }^{3}$ Lundbeck LLC, Deerfield, IL, USA. ${ }^{4}$ Pacific Northwest Statistical Consulting, Inc., Woodinville, WA, USA. ${ }^{5} \mathrm{H}$. Lundbeck A/S, Copenhagen, Denmark.

Received: 21 September 2021 Accepted: 15 November 2021

Published online: 25 November 2021

\section{References}

1. GBD (2016) Headache collaborators (2018) global, regional, and national burden of migraine and tension-type headache, 1990-2016: a systematic analysis for the global burden of disease study 2016. Lancet Neurol 17(11): 954-976. https://doi.org/10.1016/s1474-4422(18)30322-3

2. Stovner L, Hagen $K$, Jensen $R$, Katsarava Z, Lipton R, Scher A, Steiner TJ, Zwart JA (2007) The global burden of headache: a documentation of headache prevalence and disability worldwide. Cephalalgia 27(3):193-210. https://doi.org/10.1111/j.1468-2982.2007.01288.x

3. Mahmoud AN, Mentias A, Elgendy AY, Qazi A, Barakat AF, Saad M, Mohsen A, Abuzaid A, Mansoor H, Mojadidi MK, Elgendy IY (2018) Migraine and the risk of cardiovascular and cerebrovascular events: a meta-analysis of 16 cohort studies including 1152407 subjects. BMJ Open 8(3):e020498. https://doi.org/10.1136/bmjopen-2017-020498

4. Becker C, Brobert GP, Almqvist PM, Johansson S, Jick SS, Meier CR (2007) Migraine and the risk of stroke, TIA, or death in the UK (CME). Headache 47(10):1374-1384. https://doi.org/10.1111/j.1526-4610.2007.00937.x

5. Becker C, Brobert GP, Almqvist PM, Johansson S, Jick SS, Meier CR (2008) Migraine incidence, comorbidity and health resource utilization in the UK. Cephalalgia 28(1):57-64. https://doi.org/10.1111/j.1468-2982.2007.01469.x

6. Kurth T, Winter AC, Eliassen AH, Dushkes R, Mukamal KJ, Rimm EB, Willett WC, Manson JAE, Rexrode KM (2016) Migraine and risk of cardiovascular disease in women: prospective cohort study. BMJ 353:i2610. https://doi. org/10.1136/bmj.i2610

7. Kurth T, Rist PM, Ridker PM, Kotler G, Bubes V, Buring JE (2020) Association of migraine with aura and other risk factors with incident cardiovascular disease in women. JAMA 323(22):2281-2289. https://doi.org/10.1001/jama.2 020.7172

8. Ho TW, Edvinsson L, Goadsby PJ (2010) CGRP and its receptors provide new insights into migraine pathophysiology. Nat Rev Neurol 6(10):573-582 https://doi.org/10.1038/nrneurol.2010.127

9. Edvinsson L, Haanes KA, Warfvinge K, Krause DN (2018) CGRP as the target of new migraine therapies - successful translation from bench to clinic. Nat Rev Neurol 14(6):338-350. https://doi.org/10.1038/s41582-018-0003-1

10. Brain SD, Tippins JR, Morris HR, Maclntyre I, Williams TJ (1986) Potent vasodilator activity of calcitonin gene-related peptide in human skin. J Invest Dermatol 87(4):533-536. https://doi.org/10.1111/1523-1747.ep124 55620

11. AIMOVIG (2020) package insert. Amgen Inc., Thousand Oaks

12. NURTEC ODT (2021) package insert. Biohaven Pharmaceuticals, Inc., New Haven

13. UBRELVY (2021) package insert. Allergan USA, Inc., Madison

14. QULIPTA (2021) package insert. Ireland AbbVie, Dublin 
15. Homma S, Kimura T, Sakai S, K-i Y, Miyauchi Y, Aonuma K et al (2014) Calcitonin gene-related peptide protects the myocardium from ischemia induced by endothelin-1: Intravital microscopic observation and 31P-MR spectroscopic studies. Life Sci 118(2):248-254. https://doi.org/10.1016/j.lfs.2 014.02.024

16. Mulder IA, Li M, de Vries T, Qin T, Yanagisawa T, Sugimoto K et al (2020) Anti-migraine calcitonin gene-related peptide receptor antagonists worsen cerebral ischemic outcome in mice. Ann Neurol 88(4):771-784. https://doi. org/10.1002/ana.25831

17. Wackenfors A, Jarvius M, Ingemansson R, Edvinsson L, Malmsjö M (2005) Triptans induce vasoconstriction of human arteries and veins from the thoracic wall. J Cardiovasc Pharmacol 45(5):476-484. https://doi.org/10.1097/ 01.fjc.0000159639.59770.36

18. Ashina M, Goadsby PJ, Reuter U, Silberstein S, Dodick D, Rippon GA, Klatt J, Xue F, Chia V, Zhang F, Cheng S, Mikol DD (2019) Long-term safety and tolerability of erenumab: three-plus year results from a five-year open-label extension study in episodic migraine. Cephalalgia 39(11):1455-1464. https:// doi.org/10.1177/0333102419854082

19. Oakes TM, Kovacs R, Rosen N, Doty E, Kemmer P, Aurora SK, Camporeale A (2020) Evaluation of cardiovascular outcomes in adult patients with episodic or chronic migraine treated with galcanezumab: data from three phase 3 , randomized, double-blind, placebo-controlled EVOLVE-1, EVOLVE-2, and REGAIN studies. Headache 60(1):110-123. https://doi.org/10.1111/head.13 684

20. Silberstein SD, McAllister $P$, Ning $X$, Faulhaber $N$, Lang $N$, Yeung $P$ Schiemann J, Aycardi E, Cohen JM, Janka L, Yang R (2019) Safety and tolerability of fremanezumab for the prevention of migraine: a pooled analysis of phases $2 \mathrm{~b}$ and 3 clinical trials. Headache 59(6):880-890. https:// doi.org/10.1111/head.13534

21. Garcia-Martinez LF, Raport CJ, Ojala EW, Dutzar B, Anderson K, Stewart E, Kovacevich B, Baker B, Billgren J, Scalley-Kim M, Karasek C, Allison D, Latham JA (2020) Pharmacologic characterization of ALD403, a potent neutralizing humanized monoclonal antibody against the calcitonin gene-related peptide. JPET 374(1):93-103. https://doi.org/10.1124/jpet.119.264671

22. Dodick DW, Goadsby PJ, Silberstein SD, Lipton RB, Olesen J, Ashina M, Wilks K, Kudrow D, Kroll R, Kohrman B, Bargar R, Hirman J, Smith J, ALD403 study investigators (2014) Safety and efficacy of ALD403, an antibody to calcitonin gene-related peptide, for the prevention of frequent episodic migraine: a randomised, double-blind, placebo-controlled, exploratory phase 2 trial. Lancet Neurol 13(11):1100-1107. https://doi.org/10.1016/s1474-4422(14)702 09-1

23. Dodick DW, Lipton RB, Silberstein S, Goadsby PJ, Biondi D, Hirman J, Cady R, Smith J (2019) Eptinezumab for prevention of chronic migraine: a randomized phase 2b clinical trial. Cephalalgia 39(9):1075-1085. https://doi. org/10.1177/0333102419858355

24. Ashina M, Saper J, Cady R, Schaeffler B, Biondi DM, Hirman J et al (2020) Eptinezumab in episodic migraine: a randomized, double-blind, placebocontrolled study (PROMISE-1). Cephalalgia 40(3):241-254. https://doi.org/1 $0.1177 / 0333102420905132$

25. Lipton RB, Goadsby PJ, Smith J, Schaeffler BA, Biondi DM, Hirman J, Pederson S, Allan B, Cady R (2020) Efficacy and safety of eptinezumab in patients with chronic migraine. PROMISE-2. Neurology 94(13):e1365-e1377. https://doi.org/10.1212/WNL.0000000000009169

26. Dodick DW, Gottschalk C, Cady R, Hirman J, Smith J, Snapinn S (2020) Eptinezumab demonstrated efficacy in sustained prevention of episodic and chronic migraine beginning on day 1 after dosing. Headache 60(10):2220 2231. https://doi.org/10.1111/head.14007

27. Kudrow D, Cady RK, Allan B, Pederson SM, Hirman J, Mehta LR, Schaeffler BA (2021) Long-term safety and tolerability of eptinezumab in patients with chronic migraine: a 2-year, open-label, phase 3 trial. BMC Neurol 21(1):126. https://doi.org/10.1186/s12883-021-02123-w

28. Smith TR, Spierings ELH, Cady R, Hirman J, Schaeffler B, Shen V, Sperling B, Brevig T, Josiassen MK, Brunner E, Honeywell L, Mehta L (2021) Safety and tolerability of eptinezumab in patients with migraine: a pooled analysis of 5 clinical trials. J Headache Pain 22(1):16. https://doi.org/10.1186/s10194-02101227-5

29. Virani SS, Alonso A, Aparicio HJ, Benjamin EJ, Bittencourt MS, Callaway CW, Carson AP, Chamberlain AM, Cheng S, Delling FN, Elkind MSV, Evenson KR, Ferguson JF, Gupta DK, Khan SS, Kissela BM, Knutson KL, Lee CD, Lewis TT, Liu J, Loop MS, Lutsey PL, Ma J, Mackey J, Martin SS, Matchar DB, Mussolino ME, Navaneethan SD, Perak AM, Roth GA, Samad Z, Satou GM, Schroeder
EB, Shah SH, Shay CM, Stokes A, VanWagner LB, Wang NY, Tsao CW, On behalf of the American Heart Association Council on Epidemiology and Prevention Statistics Committee and Stroke Statistics Subcommittee (2021) Heart disease and stroke statistics-2021 update: a report from the American Heart Association. Circulation 143(8):e254-e743. https://doi.org/10.1161/cir. 0000000000000950

30. Deen M, Correnti E, Kamm K, Kelderman T, Papetti L, Rubio-Beltran E et al (2017) Blocking CGRP in migraine patients - a review of pros and cons. J Headache Pain 18(1):96. https://doi.org/10.1186/s10194-017-0807-1

31. Ashina M, McAllister P, Cady R, Hirman J, Ettrup A (2021) Efficacy and safety of eptinezumab in patients with migraine and self-reported aura: post hoc analysis PROMISE-1 and PROMISE-2. Headache 61(S1):123

32. Dodick D, Lipton RB, Martin V, Papademetriou $\mathrm{V}$, Rosamond W MaassenVanDenBrink A, Loutfi H, Welch KM, Goadsby PJ, Hahn S, Hutchinson S, Matchar D, Silberstein S, Smith TR, Purdy RA, Saiers J, The Triptan Cardiovascular Safety Expert Panel (2004) Consensus statement: cardiovascular safety profile of triptans (5-HT agonists) in the acute treatment of migraine. Headache 44(5):414-425. https://doi.org/10.1111/j. 526-4610.2004.04078.x

33. de Hoon JN, Willigers JM, Troost J, Struijker-Boudier HA, Van Bortel LM (2000) Vascular effects of 5-HT1B/1D-receptor agonists in patients with migraine headaches. Clin Pharmacol Ther 68(4):418-426. https://doi.org/10.1 067/mcp.2000.110502

\section{Publisher's Note}

Springer Nature remains neutral with regard to jurisdictional claims in published maps and institutional affiliations.
Ready to submit your research? Choose BMC and benefit from:

- fast, convenient online submission

- thorough peer review by experienced researchers in your field

- rapid publication on acceptance

- support for research data, including large and complex data types

- gold Open Access which fosters wider collaboration and increased citations

- maximum visibility for your research: over $100 \mathrm{M}$ website views per year

At BMC, research is always in progress.

Learn more biomedcentral.com/submissions 